\title{
Crystal structure of 4-(2-oxobenzothiazolin-3-yl)butanoic acid, $\mathrm{C}_{11} \mathrm{H}_{11} \mathrm{NO}_{3} \mathrm{~S}$
}

\author{
F. Baysen*,I R. Yağbasan' ${ }^{\mathrm{I}}$ E. Kendi ${ }^{\mathrm{II}}$, M. Aslantaşș , B. Çakır ${ }^{\mathrm{III}}$ and T. Önkol ${ }^{\mathrm{III}}$ \\ I Gazi University, Gazi Education Faculty, Department of Physics Education, Beşevler 06500, Ankara, Turkey \\ II Hecettepe University, Department of Engineering Physics, Beytepe 06532, Ankara, Turkey \\ III Gazi University, Faculty of Pharmacy, Department of Pharmaceutical Chemistry, Hipodrom 06330, Ankara, Turkey
}

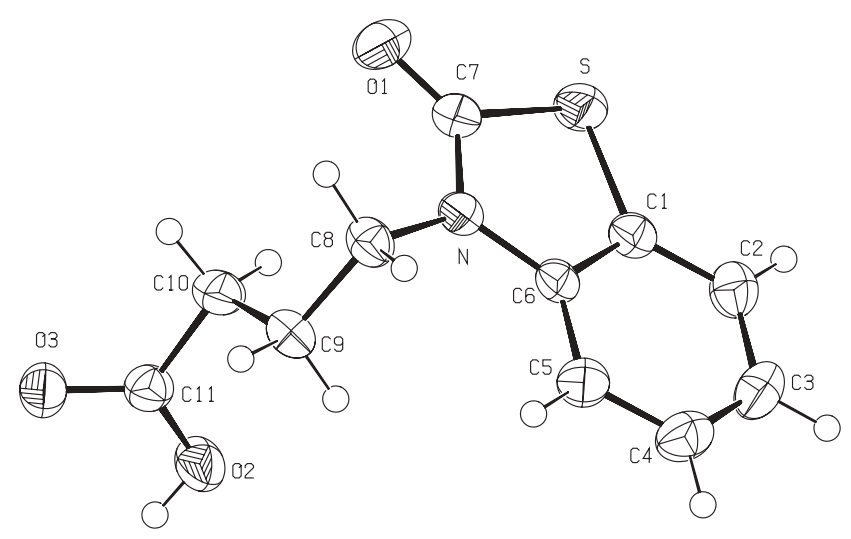

Abstract

$\mathrm{C}_{11} \mathrm{H}_{11} \mathrm{NO}_{3} \mathrm{~S}$, triclinic, $P \overline{1}$ (No. 2), $a=7.240(1) \AA, \quad b=7.495(2) \AA$, $c=10.427(1) \AA, \alpha=83.89(1)^{\circ}, \beta=85.74(1)^{\circ}, \gamma=71.16(1)^{\circ}$, $V=532.0 \AA^{3}, Z=2, R_{\mathrm{gt}}(F)=0.035, w R_{\mathrm{ref}}\left(F^{2}\right)=0.096, T=293 \mathrm{~K}$.

\section{Source of material}

For the synthesis, 10.0 mmol ethyl 4-(2-oxobenzothiazolin-3-yl)butanoate in concentrated hydrochloric acid $(50 \mathrm{ml})$ was stirred at room temperature for 2 hours, then refluxed for 4 hours. The reaction mixture was cooled, poured into $100 \mathrm{~g}$ ice-water, and stirred for 1 hour. The precipitate was collected by filtration, washed with water, dried and crystallised from water.

\section{Discussion}

The benzene ring defined by $\mathrm{C} 1-\mathrm{C} 2-\mathrm{C} 3-\mathrm{C} 4-\mathrm{C} 5-\mathrm{C} 6$ atoms is planar. Torsion angles of $\mathrm{C} 1-\mathrm{C} 2-\mathrm{C} 3-\mathrm{C} 4, \mathrm{C} 6-\mathrm{C} 5-\mathrm{C} 4-\mathrm{C} 3$ and $\mathrm{C} 1-\mathrm{C} 6-\mathrm{C} 5-\mathrm{C} 4$ are $0.4(3)^{\circ}, 0.1(3)^{\circ}$ and $0.3(3)^{\circ}$, respectively. The thiazolon ring defined by $\mathrm{C} 1-\mathrm{C} 6-\mathrm{N}-\mathrm{C} 7-\mathrm{S}$ atoms is planar. Torsion angles of $\mathrm{C} 7-\mathrm{S}-\mathrm{C} 1-\mathrm{C} 6$ and $\mathrm{C} 6-\mathrm{N}-\mathrm{C} 7-\mathrm{S}$ are $0.6(1)^{\circ}$ and $1.2(2)^{\circ}$, respectively. The dihedral angle between these two planes is $0.99(8)^{\circ}$ implying that they are co-planar. The torsion angles of $\mathrm{C} 7-\mathrm{N}-\mathrm{C} 6-\mathrm{C} 5, \mathrm{~N}-\mathrm{C} 6-\mathrm{C} 1-\mathrm{C} 2, \mathrm{C} 7-\mathrm{S}-\mathrm{C} 1-\mathrm{C} 2$ and $\mathrm{S} 2-\mathrm{C} 1-\mathrm{C} 2-\mathrm{C} 3$ are $178.8(2)^{\circ}, 179.2(2)^{\circ},-178.6(2)^{\circ}$ and $179.1(2)^{\circ}$, respectively. $\mathrm{O} 1$ and $\mathrm{C} 8$ atoms are also at this plane. Torsion angles of C6-N-C7-O1, C1-S-C7-O1, C8-N-C6-C1 and $\mathrm{C} 8-\mathrm{N}-\mathrm{C} 7-\mathrm{O} 1$ are $-179.0(2)^{\circ}, 179.2(2)^{\circ},-179.0(2)^{\circ}$ and $-0.7(3)^{\circ}$, respectively. C9 atom lies below 1.351(3) $\AA$ from thiazolon plane. Torsion angles of $\mathrm{C} 7-\mathrm{N}-\mathrm{C} 8-\mathrm{C} 9, \mathrm{C} 6-\mathrm{N}-\mathrm{C} 8-\mathrm{C} 9$ and $\mathrm{N}-\mathrm{C} 8-\mathrm{C} 9-\mathrm{C} 10$ are $103.8(2)^{\circ},-78.0(2)^{\circ}$ and $-68.5(2)^{\circ}$, re-

spectively. The bond lengths and angles in the 4-(2-oxobenzothiazolin-3-yl)butanoic acid are all in accord with similar structures in the literature [1-3]. The bond lengths of $\mathrm{C}-\mathrm{C}$ are between $1.378(3) \AA-1.522(3) \AA$. The bond lengths of $\mathrm{C} 7=\mathrm{O} 1, \mathrm{C} 11-\mathrm{O} 2$ and $\mathrm{C} 11=\mathrm{O} 3$ are 1.214(2) $\AA$, 1.262(2) $\AA$ and 1.263(2) $\AA$, respectively. The bond lengths of $\mathrm{C} 7-\mathrm{S}$ and $\mathrm{C} 1-\mathrm{S}$ are 1.782(2) $\AA$ and $1.743(2) \AA$, respectively. The bond lengths of $\mathrm{C} 6-\mathrm{N}, \mathrm{C} 7-\mathrm{N}$ and $\mathrm{C} 8-\mathrm{N}$ are $1.395(2) \AA, 1.368(2) \AA$ and $1.464(2) \AA$, respectively.

Table 1. Data collection and handling.

\section{Crystal:}

Wavelength:

$\mu$ :

Diffractometer, scan mode:

$2 \theta_{\max }$ :

$N(h k l)_{\text {measured }} N(h k l)_{\text {unique: }}$ Criterion for $I_{\mathrm{obs}}, N(h k l)_{\mathrm{gt}}$ :

$N(\text { param })_{\text {refined: }}$

Programs:

\author{
white needle, size $0.06 \times 0.27 \times 0.48 \mathrm{~mm}$ \\ Mo $K_{\alpha}$ radiation $(0.71073 \AA)$ \\ $2.94 \mathrm{~cm}^{-1}$ \\ Enraf-Nonius CAD- $4, \omega / 2 \theta$ \\ $52.58^{\circ}$ \\ 2300, 2130 \\ $I_{\mathrm{obs}}>2 \sigma\left(I_{\mathrm{obs}}\right), 1786$ \\ 147 \\ SHELXS-97 [4] SHELXL-97 [5], \\ ORTEP-III [6]
}

Table 2. Atomic coordinates and displacement parameters (in $\AA^{2}$ ).

\begin{tabular}{llllll}
\hline Atom & Site & $x$ & $y$ & $z$ & $U_{\text {iso }}$ \\
\hline $\mathrm{H}(2)$ & $2 i$ & -0.4244 & -0.0414 & 0.5929 & 0.070 \\
$\mathrm{H}(8 \mathrm{~A})$ & $2 i$ & -0.1139 & 0.5419 & 0.6207 & 0.041 \\
$\mathrm{H}(8 \mathrm{~B})$ & $2 i$ & -0.2521 & 0.5945 & 0.7436 & 0.041 \\
$\mathrm{H}(5)$ & $2 i$ & -0.3345 & 0.4424 & 0.9429 & 0.046 \\
$\mathrm{H}(4)$ & $2 i$ & -0.3352 & 0.3370 & 1.1597 & 0.053 \\
$\mathrm{H}(9 \mathrm{~A})$ & $2 i$ & -0.4118 & 0.4711 & 0.6134 & 0.042 \\
$\mathrm{H}(9 \mathrm{~B})$ & $2 i$ & -0.3442 & 0.3186 & 0.7301 & 0.042 \\
$\mathrm{H}(10 \mathrm{~A})$ & $2 i$ & -0.1531 & 0.2997 & 0.4823 & 0.048 \\
$\mathrm{H}(10 \mathrm{~B})$ & $2 i$ & -0.0717 & 0.1516 & 0.5994 & 0.048 \\
$\mathrm{H}(2 \mathrm{~A})$ & $2 i$ & 0.2404 & 0.0690 & 1.1354 & 0.048 \\
$\mathrm{H}(3)$ & $2 i$ & -0.0519 & 0.1549 & 1.2552 & 0.054 \\
\hline
\end{tabular}

\footnotetext{
* Correspondence author (e-mail: fatmac@gazi.edu.tr)
} 
Table 3. Atomic coordinates and displacement parameters (in $\AA^{2}$ ).

\begin{tabular}{llrlllllrrrr}
\hline Atom & Site & $x$ & $y$ & $z$ & $U_{11}$ & $U_{22}$ & $U_{33}$ & $U_{12}$ & $U_{13}$ \\
\hline $\mathrm{S}$ & $2 i$ & $0.32516(6)$ & $0.16590(7)$ & $0.85628(5)$ & $0.0272(2)$ & $0.0507(3)$ & $0.0421(3)$ & $-0.0099(2)$ & $-0.0009(2)$ & $-0.0008(2)$ \\
$\mathrm{O}(2)$ & $2 i$ & $-0.3456(2)$ & $0.0043(2)$ & $0.6171(1)$ & $0.0589(9)$ & $0.0509(9)$ & $0.0424(8)$ & $-0.0339(7)$ & $-0.0150(6)$ & $0.0080(6)$ \\
$\mathrm{O}(3)$ & $2 i$ & $-0.3910(2)$ & $0.1564(2)$ & $0.4210(1)$ & $0.0590(9)$ & $0.0510(8)$ & $0.0337(7)$ & $-0.0311(7)$ & $-0.0082(6)$ & $0.0022(6)$ \\
$\mathrm{O}(1)$ & $2 i$ & $0.2322(2)$ & $0.3345(2)$ & $0.6211(1)$ & $0.0448(8)$ & $0.069(1)$ & $0.0399(8)$ & $-0.0219(7)$ & $0.0058(6)$ & $0.0044(7)$ \\
$\mathrm{N}$ & $2 i$ & $-0.0148(2)$ & $0.3698(2)$ & $0.7785(1)$ & $0.0288(7)$ & $0.0354(8)$ & $0.0317(8)$ & $-0.0138(6)$ & $-0.0040(6)$ & $-0.0008(6)$ \\
$\mathrm{C}(6)$ & $2 i$ & $-0.0443(2)$ & $0.3184(2)$ & $0.9091(2)$ & $0.0309(8)$ & $0.0297(8)$ & $0.0310(8)$ & $-0.0143(7)$ & $-0.0032(7)$ & $-0.0042(7)$ \\
$\mathrm{C}(1)$ & $2 i$ & $0.1269(2)$ & $0.2062(2)$ & $0.9673(2)$ & $0.0297(8)$ & $0.0310(9)$ & $0.0354(9)$ & $-0.0108(7)$ & $-0.0014(7)$ & $-0.0053(7)$ \\
$\mathrm{C}(11)$ & $2 i$ & $-0.3165(3)$ & $0.1242(3)$ & $0.5303(2)$ & $0.0350(9)$ & $0.0347(9)$ & $0.0354(9)$ & $-0.0138(7)$ & $0.0006(7)$ & $-0.0047(7)$ \\
$\mathrm{C}(8)$ & $2 i$ & $-0.1711(3)$ & $0.4898(2)$ & $0.6966(2)$ & $0.0362(9)$ & $0.0313(9)$ & $0.0382(9)$ & $-0.0133(7)$ & $-0.0091(7)$ & $0.0015(7)$ \\
$\mathrm{C}(5)$ & $2 i$ & $-0.2193(3)$ & $0.3681(3)$ & $0.9809(2)$ & $0.0314(9)$ & $0.043(1)$ & $0.039(1)$ & $-0.0094(8)$ & $0.0010(7)$ & $-0.0052(8)$ \\
$\mathrm{C}(4)$ & $2 i$ & $-0.2187(3)$ & $0.3048(3)$ & $1.1104(2)$ & $0.043(1)$ & $0.049(1)$ & $0.042(1)$ & $-0.0148(9)$ & $0.0107(8)$ & $-0.0088(9)$ \\
$\mathrm{C}(9)$ & $2 i$ & $-0.2984(2)$ & $0.3820(3)$ & $0.6545(2)$ & $0.0300(9)$ & $0.036(1)$ & $0.041(1)$ & $-0.0119(7)$ & $-0.0069(7)$ & $-0.0038(8)$ \\
$\mathrm{C}(10)$ & $2 i$ & $-0.1901(3)$ & $0.2362(3)$ & $0.5611(2)$ & $0.039(1)$ & $0.048(1)$ & $0.042(1)$ & $-0.0243(9)$ & $0.0025(8)$ & $-0.0088(9)$ \\
$\mathrm{C}(7)$ & $2 i$ & $0.1740(3)$ & $0.3046(3)$ & $0.7310(2)$ & $0.0339(9)$ & $0.040(1)$ & $0.0361(9)$ & $-0.0184(8)$ & $-0.0007(7)$ & $-0.0032(7)$ \\
$\mathrm{C}(2)$ & $2 i$ & $0.1258(3)$ & $0.1438(3)$ & $1.0969(2)$ & $0.042(1)$ & $0.040(1)$ & $0.037(1)$ & $-0.0109(8)$ & $-0.0083(8)$ & $0.0006(8)$ \\
$\mathrm{C}(3)$ & $2 i$ & $-0.0487(3)$ & $0.1949(3)$ & $1.1678(2)$ & $0.058(1)$ & $0.047(1)$ & $0.0299(9)$ & $-0.018(1)$ & $0.0018(8)$ & $-0.0003(8)$ \\
\hline
\end{tabular}

Acknowledgment. The authors acknowledge the purchase of the CAD4 diffractometer under Grant DPT/TBAG1 of the Scientific and Technical Research Council of Turkey.

\section{References}

1. Van der Poorten, K.; Ucar, H.; Andrei, G.; Snoeck, R.; Balzarini, J.; De Clercq, E.; Pourpaert, J. H.: Synthesis and Antiviral Activity of 6-Benzoyl-Benzoxazolin-2-One and 6-Benzoyl-Benzothiazolin-2-One Derivatives. Antiviral Chem. Chemother. 10 (1999) 87-97.

2. Gokce, M.; Çakır, B.; Erol, K.; Sahin, M. F.: Synthesis and Antinociceptive Activity of [(2-Oxobenzothiazolin-3-yl)methyl]-4-Alkyl/Aryl1,2,4-Triazoline-5-Thiones. Arch. Pharm. 334 (2001) 279-283.

3. Carato, P.; Yous, S.; Depreux, P.: Synthesis of 6-Chloromethyl Benzothiazolin-2-One and of 6-Chloromethyl Benzoxazolin-2-One. Org. Prep. Proced. Int. 32 (2000) 69-74.
4. Sheldrick, G. M.: SHELXS-97. Program for the Solution of Crystal Structures. University of Göttingen, Germany 1997.

5. Sheldrick, G. M.: SHELXL-97. Program for the Refinement of Crystal Structures. University of Göttingen, Germany 1997.

6. Farrugia, L. J.: ORTEP-III for Windows. Glasgow University, Department of Chemistry, Glasgow G12 8QQ, Scotland, UK 1998. 